\title{
Embolization of Dural Arteriovenous Fistula of the Sinus Confluence with Onyx using Dual-Lumen Microballoon Catheter: A Case Report
}

\author{
Yosuke Kawamura, Tomoji Takigawa, Akio Hyodo and Kensuke Suzuki* \\ *Department of Neurosurgery, Dokkyo Medical University Saitama Medical Center, Japan
}

Received: June 20, 2018; Published: June 27, 2018

*Corresponding author: Kensuke Suzuki, Department of Neurosurgery, Dokkyo Medical University Saitama Medical Center, Saitama, Japan

Abstract

Dural arteriovenous fistula (DAVF) is classically defined as abnormal arteriovenous connections between an arterial feeder and a dural venous sinus or leptomeningeal vein with the channels located within the dural leaflets [1]. Onyx is a non-adhesive liquid embolic agent and does not polymerize. The dimethyl sulfoxide can diffuse under aqueous conditions and occlude mechanically the feeders [2]. We describe transarterial embolization with dual-lumen balloon microcatheter with Onyx (Medtronic, Minneapolis, MN, USA) for dural arteriovenous fistula (DAVF) of sinus confluence.

\section{Case Presentation}

A 73-year-old-man presented with right tinnitus one year before hospitalization. And his cognitive function gradually declined. He was performed MRI, MRA to check dementia in another neurosurgical clinic. It showed abnormal signal voids. He was referred to our hospital and performed an angiography. Angiography demonstrated a DAVF adjacent to the confluence of sinuses, which supplied by bilateral occipital artery. The fistula drained into an isolated transverse sinus, and to a straight sinus, Rosenthal vein, superficial sylvian vein with cortical venous reflux (Figures 1 \& 2A). Transarterial embolization (TAE) was performed through the transfemoral approach. The procedure was performed under general anesthesia. A 4French(Fr.) catheter was introduced to the right external carotid artery for control angiography. A Flexor-Shuttle-Guiding Sheath 7Fr. (COOK Medical, Bloomington, IN, USA) was placed into the left external carotid artery. A 6Fr. guiding catheter (FUBUKI, Asahi Intecc, Aichi, Japan) was placed into left occipital artery over a 0.035 -inch outer diameter guidewire (Radifocus, Terumo, Tokyo, Japan).

A dual-lumen microballoon catheter (ScepterXC, Microvention, Tustin, California, USA) was navigated to the distal of occipital artery over a 0.014-inch outer diameter microguidewire (CHIKAI, Asahi Intecc, Aichi, Japan) with a 4.2Fr. guiding catheter (FUBUKI, Asahi Intecc, Aichi, Japan), and the balloon was inflated. And we injected Onyx 18 into the shunt point and infiltrated into the feeding arteries. A total of $2.96 \mathrm{cc}$ of Onyx was injected over a period of
17 minutes, after which the DAVF was completely occluded (Figure $2 \mathrm{~B}$ ). Total procedure time was 1 hour 34 minutes. The $4.2 \mathrm{Fr}$. guiding catheter was navigated into the occipital artery as distal as possible for successful removal of Scepter XC. The final angiograms presented the occlusion of DAVF (Figure 3). No complications associated with the procedure were evident. And his symptom was gradually better.

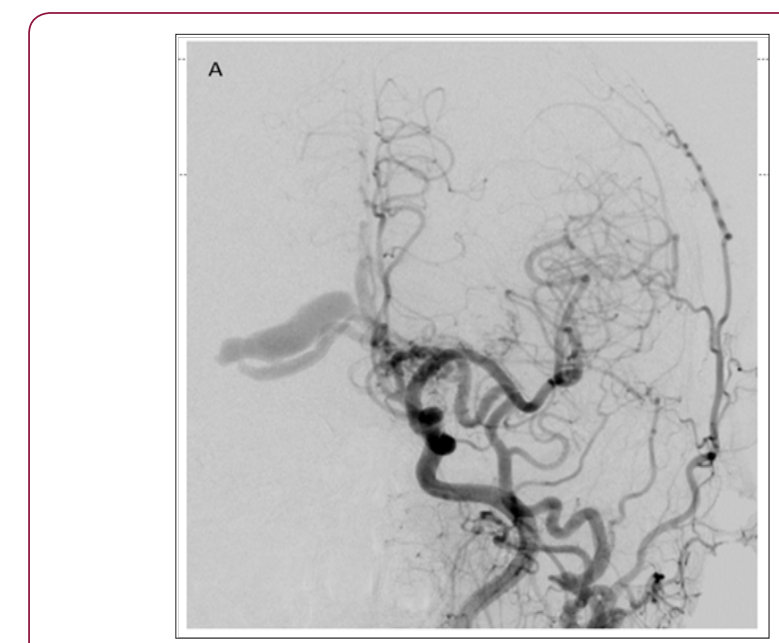

Figure 1: Left Common carotid artery angiogram an anterioposterior view (A) Showing the DAVF adjacent to the confluence of sinuses. 


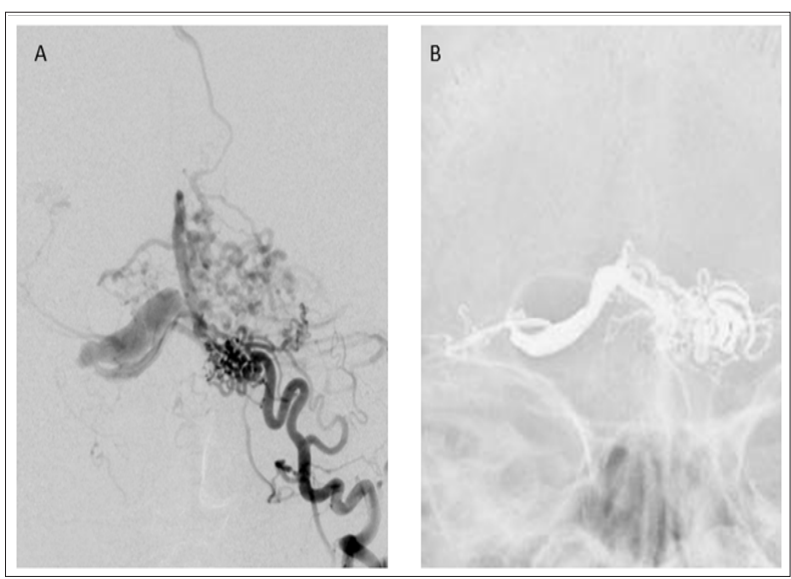

Figure 2: Left occipital artery angiogram an anteroposterior view (A). The supply to the fistula is mainly from left occipital artery. The fistula drains into a right isolation transverse sinus and to straight sinus, cortical vein. Angiographies Show the anteriovenous fistulous shunt is in the adjacent to the confluence of sinus. Note the cast of the Oynx at the end of injection(B).

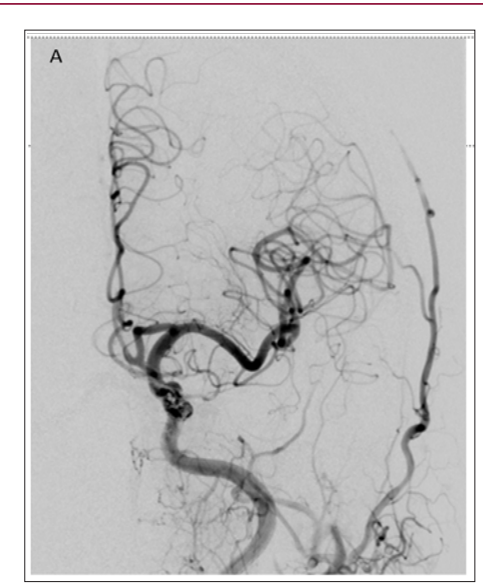

Figure 3: Left Common carotid artery angiogram, an anterioposterior view (A) showing the complete obliteration of the DAVF.

\section{Discussion}

The definitive treatment of DAVF requires the occlusion of the arteriovenous shunt without interfering with the normal venous drains. Transvenous approach is not always possible in cases with difficult anatomy such as isolated sinus. On the other hand, it is reported that transarterial Onyx embolization is particularly useful in Cognard type III and IV [3]. We presented the safe and effective transarterial embolization with a dual-lumen balloon microcatheter with Onyx for DAVF of the sinus confluence. Onyx injection needs long fluoroscopy times and radiation exposure [4]. The dual-lumen balloon microcatheter makes Onyx plug easy and shorten the procedure times and reduce radiation exposure [5]. In our case, we could perform the procedure in a short time, total procedure time was 1 hour 34 minutes. We use a 4.2Fr. guiding catheter for supporting the dual-lumen balloon microcatheter and for microcatheter removal from Onyx cast. Our supporting guiding system is particularly useful for Onyx embolization.

This is the useful report about the effective and safe technique of transarterial embolization using a dual-lumen balloon microcatheter with Onyx for DAVF of the sinus confluence. It is one of the treatment options for DAVF.

\section{References}

1. Rhaul G, Shigeru M, Noriaki M, Izumi T, Natio T, et al. (2013) A unique type of dural arteriovenous fistula at confluence of sinuses treated with endovascular embolization: a case report. Neurointerv 8(1): 34-40.

2. Jian W, HuanCheng WU, Wei-Wei W, Zhao HS, Dao RN, et al. (2016) Trigeminal cardiac reflex caused by Onyx embolization of intracranial dural arteriovenous fistula. J TrukNeurosug26(3): 325-330.

3. Chao BL, Feng CC, Michael MHT, Lin CJ, Wu HM, et al. (2014) Transarterial Onyx embolization of intracranial dural arteriovenous fistulas: A single center experience. J Chin Med Assoc77: 184-189.

4. Velat GJ, ReaveyCantwell JF, Sistrom C, David Smullen, Gregory LF, et al. (2008) Comparison of N-butyl cyanoacrylate and Onyx for the embolization of intracranial arteriovenous malformations: analysis of fluoroscopy and procedure times. Neurosurgery63: ONS73-78.

5. Guilherme D, Italo L, Mario M (2014) Endovascular treatment of dural arteriovenous fistulas using dual lumen balloon microcatheter: Technical aspects and results. Clinical Neurology and Neurosurgery 117: 22-27.
CC This work is licensed under Creative Commons Attribution 4.0 License

Submission Link: https://biomedres.us/submit-manuscript.php

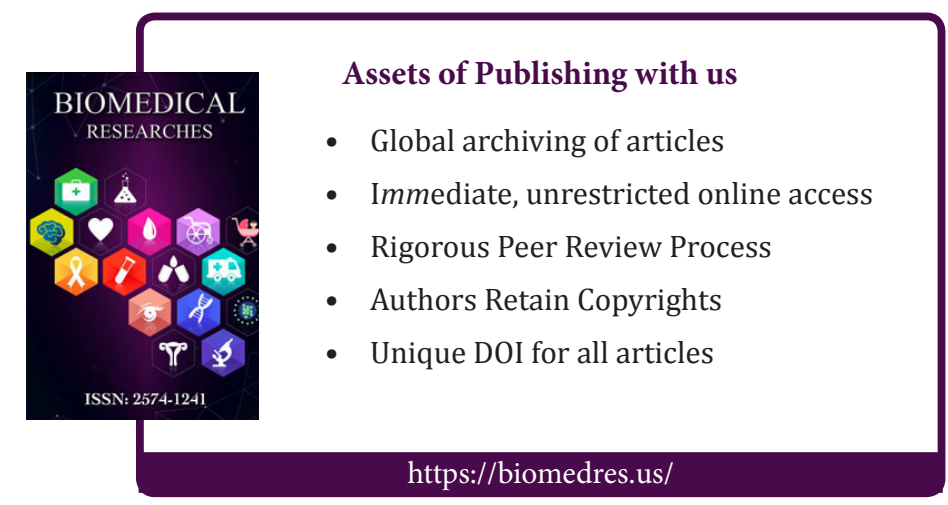

